DEVELOPMENTAL BIOLOGY

\section{How to get your bearings}

As development progresses, cells frequently need to assess positional information if organ and tissue formation are to occur normally. Exactly how cells monitor their position and integrate the relevant signals they receive is not well understood. Costa and Shaw now show that in Arabidopsis thaliana root epidermis, position-dependent cell-fate switching depends on alternative states of chromatin organization at a specific locus. The switch is rapid, ensuring a high level of developmental plasticity.

The root epidermis of Arabidopsis is made up of rows of hair and nonhair cells. This pattern is established in embryogenesis and propagated in the seedling root, but rather than being simply lineage-dependent, it relies on both positional information and the activity of GLABRA2 - a homeodomain transcription factor - in the future non-hair cells. Using three-dimensional fluorescence in situ hybridization (3D-FISH) of intact epidermis, the authors found that in hair cells, where GLABRA2 is not expressed, chromatin around this locus is in a closed conformation; the converse was true for non-hair cells.

To explore the link between chromatin, GLABRA2 expression and cell-fate specification, Costa and Shaw turned to A. thaliana mutants. Position-dependent expression of GLABRA2 is controlled by WEREWOLF (WER) and CAPRICE (CPC), two Myb proteins. 3D-FISH and phenotypic analysis of wer and $c p c$ mutants indicated that the closed chromatin conformation in hair cells requires $\mathrm{CPC}$, but that open chromatin at the GLABRA2 locus requires neither GLABRA2 expression nor cell-fate specification. Propagation of positional cues requires correct chromatin assembly - in fasciata 2 (fas2) mutants, in which chromatinassembly factor- 1 is defective, the wild-type epidermal pattern is not established and GLABRA2 is ectopically expressed.

Chromatin organization around GLABRA2 is not irreversibly fixed. The authors used clonal analysis to expose cells of the same clonal origin to different positional cues and found that the GLABRA2 locus was remodelled in response to the new position. The remodelling was surprisingly rapid - careful FISH analysis of two-cell clones revealed that the chromatin state is reset during mitosis and respecified in G1. So the cell-fate switch can take place without the need for completion of the cell cycle.

Costa and Shaw provide an elegant demonstration of how chromatin organization integrates positional information with gene-expression states. In the future, knowing the identity of the upstream players that are involved in this process will reveal how external cues are translated into changes at the chromatin level.

Magdalena Skipper
ORIGINAL RESEARCH PAPER Costa, S. $\varepsilon$ Shaw, P. Chromatin organization and cell fate switch respond to positional information in Arabidopsis. Nature 18 December 2005 doi:10.1038/nature04269

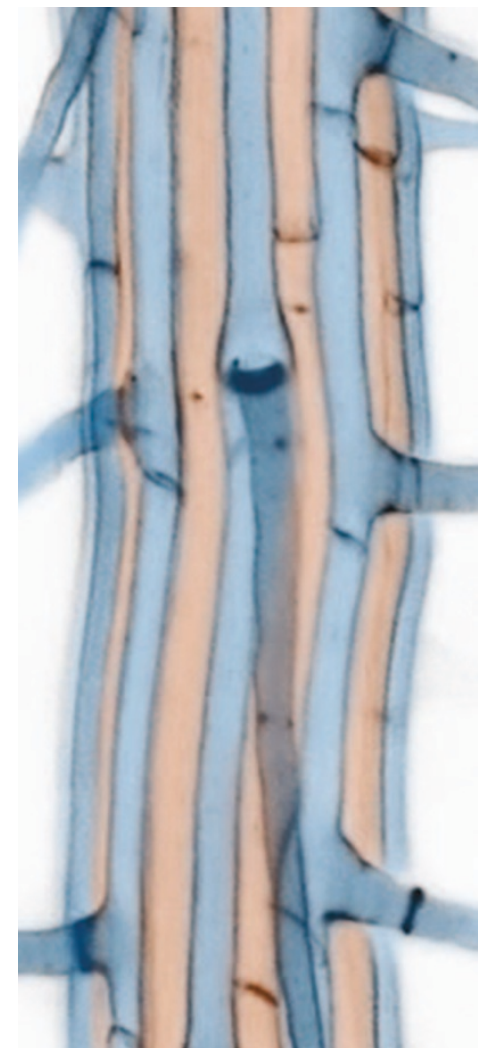

Image courtesy of Silvia Costa and Peter Shaw. 